\title{
Respuesta de plántulas de Cucurbita pepo var. Scallop a la fertilización fosfórica
}

\section{Cucurbita pepo var. Scallop seedlings response to phosphoric fertilization}

ANA MARÍA CASTAGNINO'

KARINA ELIZABETH DÍAZ1

PATRICIA SASTRE V. ${ }^{1}$

MIGUEL NAVARRO D. ${ }^{1}$

La familia Cucurbitaceae constituye un grupo vegetal muy importante desde el punto de vista económico, nutricional y cultural. Foto: A.M. Salama*

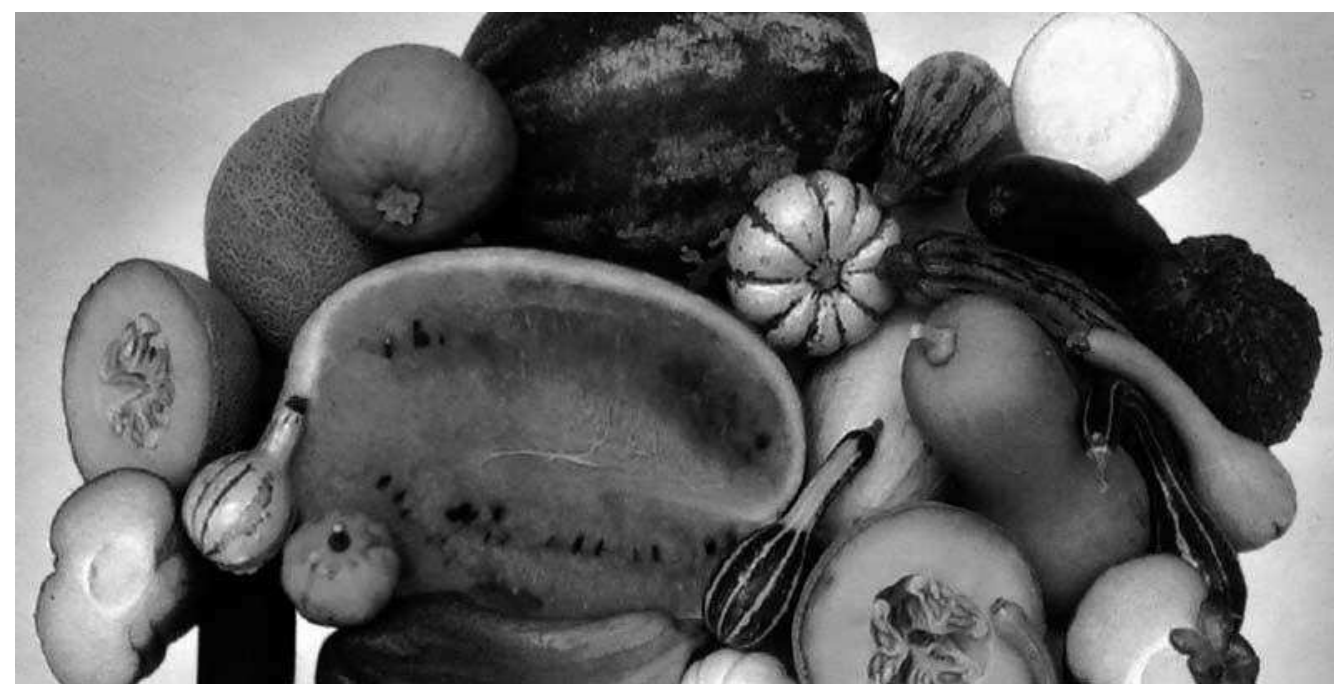

\section{RESUMEN}

Cucurbita pepo var. Scallop es un cultivo de clima cálido muy vigoroso, que se caracteriza por la forma original de sus frutos con borde festoneado. El suministro de nutrientes minerales es uno de los factores ambientales que más influyen sobre su crecimiento. El objetivo del presente trabajo consistió en evaluar el efecto de distintas dosis de fósforo sobre el rendimiento. Este trabajo se realizó en la Chacra Experimental de la Facultad de Agronomía de la UNCPBA, en Azul (Argentina). Los tratamientos realizados fueron: testigo sin fertilización, 100, 200, 300, 400 y $500 \mathrm{~kg}$ de fosfato diamónico. El cultivo se inició mediante plantines con cepellón, trasplantados, el 15/11, en camellones cubiertos con mulching naranja y en condiciones de secano, con una densidad de 20.000 plantas/ha ( $2 \times 0,5 \mathrm{~m}$ ). La superficie total del ensayo fue de $1.488 \mathrm{~m}^{2}$, diseñado bajo un modelo de bloques completamente aleatorizados. El rendimiento promedio general fue de $48.410 \mathrm{~kg} \cdot \mathrm{ha}^{-1}$. El mayor rendimiento total en tha ${ }^{-1}$ se logró con la aplicación de la dosis de $300 \mathrm{~kg} \cdot \mathrm{ha}^{-1}$ de fosfato diamónico. El número de frutos obtenido en los tres tratamientos intermedios, presentó un comportamiento muy similar pero diferenciado de las restantes dosis de 100 y $500 \mathrm{~kg} \cdot \mathrm{ha}^{-1}$.

* Tomado de: Salama, A.M. 2006. Las cucurbitáceas: importancia económica, bioquímica y medicinal. Departamento de Farmacea, Facultad de Ciencias, Universidad Nacional de Colombia, Bogotá. 196 p.

1 CRESCA, Centro Regional de Estudio de Cadenas Agroalimentarias y Programa Institucional de Alimentos, Facultad de Agronomía, Universidad Nacional del Centro de la Provincia de Buenos Aires, Azul (Argentina).amc@faa.unicen.edu.ar 


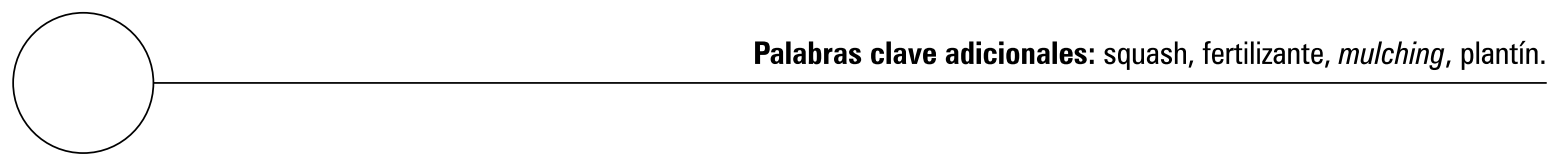

\section{ABSTRACT}

Cucurbita pepo var. Scallop is a very vigorous crop from warm climate areas which outstands for the particular shape of its fruits with a festooned edge. Mineral nutrient supply is one of the environmental factors that influence mostly its growth. The aim of this paper was to evaluate the effect of different doses of phosphorus on yield. This trial was carried out in the Experimental Unit of Agronomy Faculty - UNCPBA, in Azul in the Argentinian Wet Pampa. The treatments were: control without fertilization; 100; 200; 300; 400 and $500 \mathrm{~kg}$ of ammonium phosphate. The crop started from seedlings with root ball which were transplanted on $15 / 11$, on camlets covered with orange mulching, not irrigated and with a density of 20,000 plants/ha $(2 \times 0.5 \mathrm{~m})$. The trial covered a total area of $1.488 \mathrm{~m}^{2}$. The trial was designed under a model of completely randomized blocks. General average yield was $48,410 \mathrm{~kg} \cdot \mathrm{ha}^{-1}$. The greatest total yield in $\mathrm{t} \cdot \mathrm{ha}^{-1}$ was obtained with the application of $300 \mathrm{~kg} \cdot \mathrm{ha}^{-1}$ of ammonium phosphate. The number of fruits obtained in treatments 3, 4 and 5 showed similar response, but differed from that of the other doses of 100 and $500 \mathrm{~kg} \cdot \mathrm{ha}^{-1}$.

Additional key words: squash, fertilizer, mulching, seedlings.

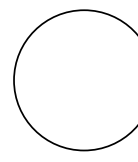

INTRODUCCIÓN

Cucurbita pepo var. Scallop, comúnmente denominados 'Zapallitos Ovni', 'Zapallitos Scallopini' y recientemente denominados 'Patty Pan', es un cultivo muy vigoroso, que se caracteriza por la forma original de sus frutos, aplanados con borde ondulado o festoneado, similares a un ostión, de varios colores (verdes, blancos, amarillos). Algunos cultivares son 'Golden Bush Scallop', 'Benning's Green Tint' y 'Patty Pan'. El interior de los frutos es de color amarillo pálido y de textura más compacta que los zapallitos redondos de tronco.

En otros países también se le conoce con diversos nombres: 'Cymling', 'Scallop Squash', 'Custard Marrow', o 'Custard Squash'. Su nombre francés 'Pâtisson', deriva de una palabra provenzal para un pastel hecho en un molde festoneado. En Latinoamérica se le cultiva comercialmente desde hace pocos años y constituye una alternativa productiva interesante y novedosa, principalmente para el mercado de las denominadas hortalizas 'Baby' y por su aporte de magnesio, niacina y vitaminas A y C, sumado a su escaso aporte energético (20-30 calorías/porción).

Es un cultivo de clima cálido, cuyo rango óptimo de temperaturas para la germinación es de 24 a $34^{\circ} \mathrm{C}$, siendo la mínima $15,6^{\circ} \mathrm{C}$. Para la floración requiere 20 a $25^{\circ} \mathrm{C}$, mientras que 25 a $30^{\circ} \mathrm{C}$ para el desarrollo del cultivo. La temperatura a nivel de las raíces durante el período de crecimiento debe ser superior a $10^{\circ} \mathrm{C}$. No son plantas guiadoras y conforman una planta compacta, tipo arbustiva. La mayoría de los trabajos encontrados sobre esta especie fueron realizados en Haifa, Israel (Paris y Nelson, 1986; Paris, 1988; 1992). 
El suministro de nutrientes y minerales es uno de los factores ambientales que más influyen sobre el crecimiento de las plantas, el desarrollo de un sistema radicular denso y profundo que les permita acceder al uso de un mayor volumen de agua del suelo, amortiguando los efectos de sequías intermitentes (Darwich, 1994) en cultivos de secano como el presente.

El fósforo, uno de los principales macronutrientes, está presente en la mayoría de los suelos del mundo variando entre 100 y $2.450 \mathrm{~kg} \cdot \mathrm{ha}^{-1}$ para la capa arable $0-20 \mathrm{~cm}$, con una media de aproximadamente $1.000 \mathrm{~kg} \cdot \mathrm{ha}$ (Darwich, 1994). Los suelos de la zona centro de la provincia de Buenos Aires, en Argentina poseen bajo contenido de fósforo disponible para las plantas, deficiencia que se ha agravado durante los últimos años debido a la falta de reposición y a lo extraído por sucesivas cosechas (Darwich, 1989 y 1994), por lo que se torna indispensable su aporte. Según el mismo autor, la cuenca del Arroyo Azul, en la que se efectúa este trabajo, estaría comprendida en la denominada "Areal", en la que predominan los suelos deficientes en fósforo.

Existe una estrecha relación entre el contenido de fósforo disponible en el suelo y el aumento de eficiencia por la colocación del fertilizante en bandas. (Darwich, 1989). Aplicaciones de fosfato diamónico colocado a los costados y por debajo de la semillas o plántulas resultan más efectivas que las realizadas al voleo; ya que se facilita la absorción, aumentando la eficiencia de la fertilización y el rendimiento del cultivo (Mullins et al., 1992; Martínez, 1997).

La absorción de fósforo presenta una curva similar a la de acumulación de materia seca, con una fase linear que inicia con el engrosamiento de los primeros frutos cuajados y termina con la maduración (Quattrucci, 2001).

El empleo de macetas, vasos o speedlings con celdas de más de $5 \mathrm{~cm}$ de diámetro para el inicio de los cultivos se realiza en aquellas especies que necesitan de un mayor espacio de crecimiento y está esencialmente destinada a hortalizas de fruto (Cucurbitáceas y Solanáceas) logrando mayor precocidad en la producción, como para soportar condiciones ambientales adversas, cultivos homogéneos y potencialmente más productivos (Giustiniani et al., 2001).

El empleo de medidores cuánticos de la radiación permite predecir el comportamiento del cultivo, ante las distintas variables estudiadas desde los primeros estadios del mismo, por lo que constituye una técnica complementaria a determinaciones posteriores.

El mulching plástico ejerce su acción sobre la humedad, la temperatura, la estructura del terreno, las malezas, los elementos nutritivos y la acción microbiológica. Respecto de la humedad del suelo se limitan las pérdidas por evaporación y en consecuencia las reservas hídricas son mantenidas por más tiempo. A causa de la naturaleza impermeable del plástico, el agua de lluvia se acumula en los laterales del mulching desde donde por imbibición e infiltración lateral penetra en el terreno. Respecto al terreno descubierto se pueden relevar incrementos de temperatura de entre 0,5 a $7^{\circ} \mathrm{C}$. Además, la estructura del terreno se puede conservar por mayor tiempo, gracias a la protección de los agentes atmosféricos (Castagnino et al., 2006).

El objetivo del presente trabajo consistió en evaluar el efecto de distintas dosis de fosfato diamónico sobre el rendimiento de 'Zapallitos Ovni', mediante las variables: intercepción de radiación, rendimiento en tha ${ }^{-1}$ y número de frutos/ha.

\section{MATERIALES Y MÉTODOS}

Este trabajo se realizó en la chacra experimental de la Facultad de Agronomía, sobre la Ruta Nacional $\mathrm{N}^{\circ} 3, \mathrm{~km} \mathrm{305}$, en la ciudad de Azul, a los $36^{\circ} 45^{\prime} \mathrm{S}$ y $59^{\circ}$ y $57^{\prime} \mathrm{W}$ a los $137 \mathrm{msnm}$, en La Provincia de Buenos Aires, sobre un suelo argiudol típico. El período medio libre de heladas es de 
208 días y el régimen de precipitaciones presenta una distribución normal, con una media anual de $850,4 \mathrm{~mm}$, concentrándose la mayor proporción en el período primavero-estival.

La preparación del lote consistió en arada y varias pasadas de rastra de discos cruzadas y se complementó con motocultivador. Luego se conformaron camellones de $80 \mathrm{~cm}$ de ancho, y posteriormente se procedió a aplicar las distintas dosis de fertilizante previstas. Este ensayo se realizó en condiciones de secano.

Los tratamientos realizados fueron testigo (1) y aplicaciones en banda de $\left(\mathrm{NH}_{4}\right)_{2} \mathrm{HPO}_{4}$ fosfato diamónico (18-46-0): 100 (2), 200 (3), 300 (4), $400(5)$ y $500(6) \mathrm{kg} \cdot \mathrm{ha}^{-1}$.

Una vez incorporado el fertilizante se procedió a cubrir los camellones con mulching naranja de un metro de ancho y de $50 \mathrm{~cm}$ de espesor. Los plantines sembrados en bandejas de cartón, tipo speedling el día 28/10 y mantenidos hasta el momento del trasplante en un túnel, se trasplantaron el 15/11, con una densidad de 20.000 plantas/ha $(2 \times 0,5 \mathrm{~m})$.

La semilla de 'Zapallitos Ovni' utilizada fue 'Rogers Sandoz Seed'. La superficie total del ensayo fue: $1.488 \mathrm{~m}^{2}$, siendo el largo total de $62 \mathrm{~m}$ y el ancho de $24 \mathrm{~m}$. Los caminos fueron de $4 \mathrm{~m}$ de ancho y las cabeceras de $5 \mathrm{~m}$. Cada parcela tuvo una superficie evaluable de $20 \mathrm{~m}^{2}$. Los dos surcos exteriores fueron considerados borde. El número total de parcelas por bloque fue de $8 \mathrm{y}$ total del ensayo de 32 .

Se realizaron controles de malezas químicos, manuales y mecánicos. Previo a la realización de los camellones se aplicó Trifluralina a razón de $2 \mathrm{~L}^{2} \mathrm{ha}^{-1} \mathrm{y}$ se incorporó con el motocultivador. Luego del trasplante se aplicó Naptalam a razón de $15 \mathrm{~L} \cdot \mathrm{ha}^{-1}$, en los entresurcos. Durante el ciclo de cultivo se efectuaron controles mecánicos con motocultivador en los entresurcos y manuales en los bordes de los camellones.
Se efectuaron controles preventivos y curativos de insectos con Cipermetrina; y de enfermedades con el fungicida Benomyl. Se midió una vez al día la radiación interceptada, con un sensor cuántico lineal LI-COR 1000 en el mediodía solar (entre las 11:00 y 13:00 h), con un máximo de un 20\% de nubosidad. El mismo se introdujo por debajo del canopeo del cultivo determinando radiación interceptada y por encima la radiación total incidente.

Se utilizó un diseño de bloques completamente aleatorizados con 5 tratamientos y un testigo. Los datos se analizaron bajo un modelo de bloques con parcelas divididas en el tiempo donde los tratamientos fueron la parcela principal y las cosechas constituyeron las subparcelas. Previo al análisis de la varianza se realizaron test de esfericidad y normalidad a fin de comprobar el cumplimiento de los supuestos requeridos por el modelo. Para la comparación de medias se usó el test DMS. Se analizaron variables como rendimiento en tha ${ }^{-1}$, número de frutos por ha y peso promedio por unidad.

\section{RESULTADOS}

El rendimiento promedio general del ensayo fue de $48.410 \mathrm{~kg} \cdot \mathrm{ha}^{-1}(s= \pm 3,760)$. Esta producción se obtuvo a lo largo de once cosechas realizadas durante el período comprendido entre el 29 de diciembre y el 26 de febrero, no habiéndose detectado diferencias entre los tratamientos durante cada cosecha.

En el análisis efectuado sobre la variable rendimiento total medida en tha ${ }^{-1}$, se detectan diferencias significativas entre las dosis aplicadas. De la comparación entre las medias de los tratamientos surgen tres grupos superpuestos: a) el compuesto por los tratamientos: $3,4,5$ y 6 ; b) el formado por los tratamientos $1,2,3,5$ y $6 ; \mathrm{y} \mathrm{c}$ ) el constituido por los tratamientos $1,2,3$ y 5 . El rendimiento promedio obtenido con la aplicación de $\left(\mathrm{NH}_{4}\right)_{2}$ $\mathrm{HPO}_{4}$ fosfato diamónico (18-46-0) a $300 \mathrm{~kg} \cdot \mathrm{ha}^{-1}$ supera al que se obtiene con la dosis $100 \mathrm{~kg} \cdot \mathrm{ha}^{-1}$ $\mathrm{y}$ al rendimiento promedio del cultivo testigo. Las 
diferencias entre los restantes promedios no son significativamente diferentes. Sin embargo se observa una tendencia que hace pensar que la dosis óptima podría encontrarse cercana a la dosis correspondiente al tratamiento 4 , ya que tanto para dosis superiores como para inferiores a esta, las tendencias marcan una disminución de los rendimientos medidos en tha ${ }^{-1}$ (tabla 1 ).

El rendimiento promedio por ha expresado en frutos, resultó de 108.611 frutos/ha $(s= \pm 7,187)$.

Respecto a la variable número de frutos del análisis de la variancia surge que existen diferencias significativas entre los tratamientos considerados. De la comparación de las medias es posible individualizar nuevamente tres grupos superpuestos constituidos por los tratamientos: a) 3 , 4,5, y 6; b) 1 y 6; c) 1 y 2 . Con la aplicación de las dosis 3, 4 y 5 se obtiene mayor número de frutos que los que producen la aplicación de la dosis 2 y el cultivo sin tratar. Con la aplicación de la dosis 6 se obtiene mayor cantidad de frutos que con la aplicación de la dosis 2 . Las restantes medias no resultaron significativamente diferentes. Al analizar esta variable nuevamente se observa la tendencia marcada anteriormente.

\section{DISCUSIÓN}

En el cultivo de esta cucurbitácea existe una fase lineal de intenso crecimiento durante la cual se

Tabla 1. Influencia de la fertilización sobre el rendimiento en Cucúrbita pepo var. Scallop.

\begin{tabular}{|c|c|c|c|}
\hline Tratamiento & $\begin{array}{c}\text { Rendimiento } \\
\text { t·ha- }{ }^{-}\end{array}$ & $\begin{array}{c}\text { Número } \\
\text { frutos/ha }\end{array}$ & $\begin{array}{c}\text { Peso unitario } \\
\text { g/fruto }\end{array}$ \\
\hline 4 & $54,25 \mathrm{a}^{*}$ & $118.333 \mathrm{a}$ & 458 \\
\hline 6 & $51,39 \mathrm{ab}$ & $108.833 \mathrm{ab}$ & 472 \\
\hline 3 & $48,53 \mathrm{abc}$ & $119.000 \mathrm{a}$ & 408 \\
\hline 5 & $48,19 \mathrm{abc}$ & $115.400 \mathrm{a}$ & 418 \\
\hline 1 & $44,50 \mathrm{bc}$ & $97.500 \mathrm{bc}$ & 456 \\
\hline 2 & $42,55 \mathrm{bc}$ & $92.550 \mathrm{c}$ & 460 \\
\hline
\end{tabular}

Promedios con letras distintas son significativamente diferentes según la prueba de DMS $(P \leq 0,05)$ registran los máximos valores de acumulación de sustancia seca y absorción de macronutrientes (Quattrucci, 2001). Los mayores rendimientos obtenidos se explicarían por el desarrollo de un sistema radicular más denso y profundo que en los restantes tratamientos (Darwich, 1989). El aporte de fosfato diamónico (18-46-0), incrementa el área foliar y la acumulación de materia seca en las hojas, lo que quedó evidenciado en las mediciones de intercepción realizadas durante el período vegetativo que resultaron ser superiores para los tratamientos 3, 4 y 5 . Respecto de las mediciones de intercepción realizadas en el período 24/12 - 14/01, se destacan las correspondientes al tratamiento 4 (figura 1 ).

Debido a condiciones climáticas adversas se demoró la realización de la décima cosecha respecto de las demás. Esto motivó que los frutos incrementaran su tamaño promedio y por ende se lograra un mayor rendimiento total durante la misma.

El uso del mulching permitió que el cultivo se desarrollara en todo momento dentro del rango óptimo de temperatura de suelo apropiado para esta especie. De no haberse utilizado esta técnica de protección las plantas habrían estado expuestas a temperaturas de suelo inadecuadas para su normal crecimiento durante el período de prefloración. Esta técnica de defensa permitió aumentar aproximadamente en $5^{\circ} \mathrm{C}$ las temperaturas del suelo medidas, a 5 y a $10 \mathrm{~cm}$ de profundidad, respecto de las mediciones efectuadas en el suelo desnudo (figura 2), lo que posiblemente contribuyó a incrementar el rendimiento en número de frutos y tha ${ }^{-1}$, tal como el encontrado por Cantamuto (1997); Castillo (1998) y Alvarado (1998).

Debido a las fluctuaciones de la temperatura del suelo existe un cambio o variación estacional en la disponibilidad de fosfatos dado por el aporte de la mineralización. Esto conduce a que en épocas frías se encuentren valores mínimos de fósforo disponible en el suelo y que los mismos alcancen un máximo hacia finales de verano (Darwich, 1989). El incremento de la temperatura y 


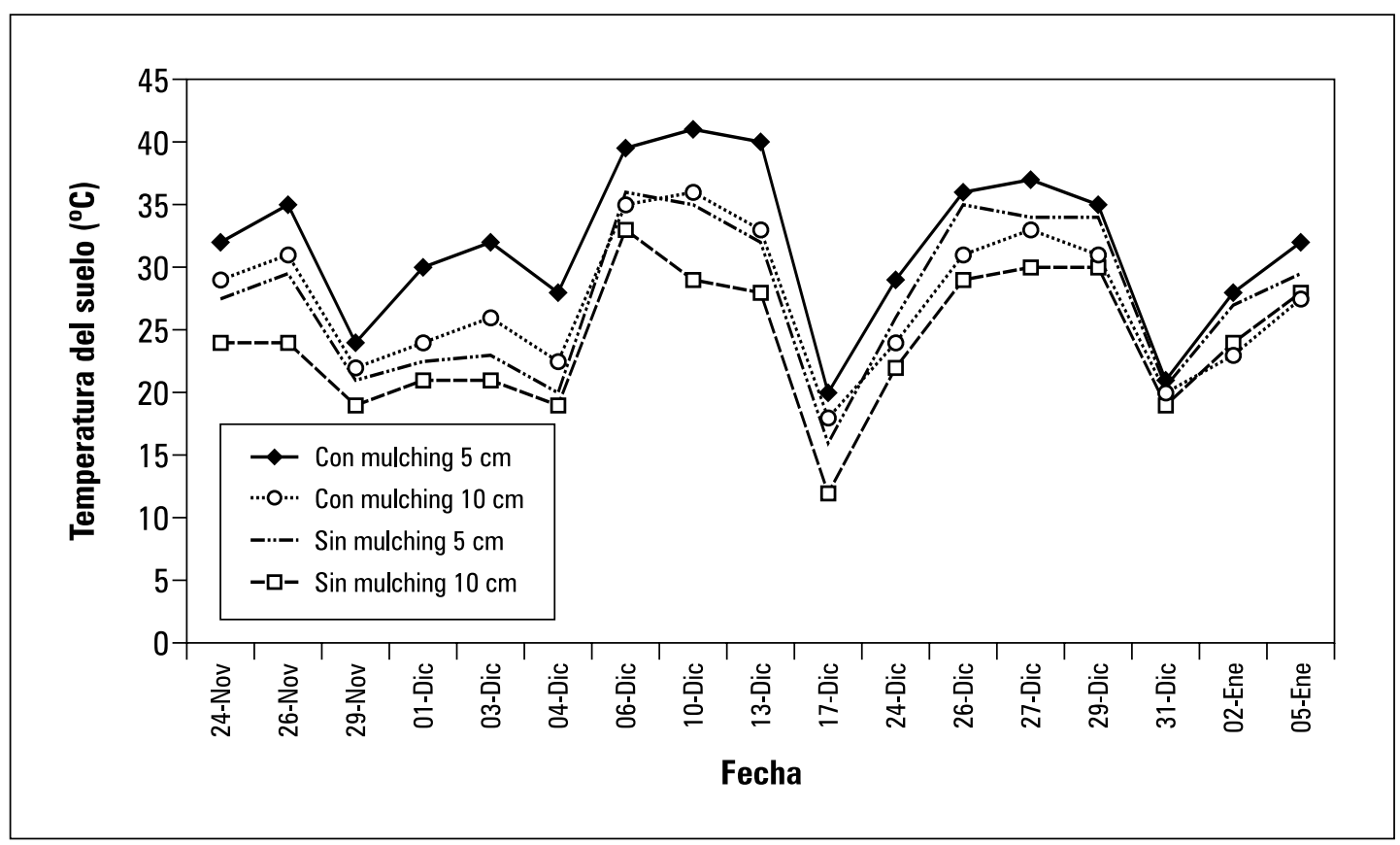

Figura 1. Temperatura del suelo, con y sin mulching a diferentes profundidades durante el período de cultivo en Cucurbita pepo var. Scallop.

la disminución de las fluctuaciones de la misma por efecto del mulching contribuyó a optimizar la disponibilidad de fósforo (Darwich, 1983 y 1994).

Desde hace varios años, existe en el sector hortícola una continua transformación hacia sistemas de cultivo orientados al mejoramiento cualicuantitativo de la producción, al aumento de la eficiencia en el uso del agua, de fertilizantes, de la superficie protegida y al respeto del ambiente. En ese contexto, el empleo de plantines con cepelón para el inicio de los cultivos hortícolas constituye una de las innovaciones más importantes de las últimas décadas (Castagnino et al., 1999) y el primer paso hacia un sistema que coloca la empresa hortícola en grado de optimizar la calidad de las producciones y los costos de producción, producir respetando el ambiente y la salud; además de una adecuada programación de las producciones (Trentini, 1996). Este sistema permite también anticipar el inicio de los cultivos a campo en zonas de riego de heladas tardías, como en el centro de la Provincia de Buenos Aires.
Las ventajas del uso de mulching plástico para la producción de hortalizas se reconoce desde 1950 (Emmert, 1957). El mulching plástico eleva la temperatura del suelo, promoviendo un desarrollo más rápido del cultivo y una cosecha más temprana (Loy y Wells, 1990).

El empleo de mulching naranja brindó al cultivo mejores condiciones de humedad y temperatura del suelo, además de evitar la presencia de malezas en las hileras. En inicio mediante el sistema de almácigo y trasplante con cepellón posibilitó la ausencia de fallas en el cultivo y la uniformidad de las plantas a lo largo del ciclo.

\section{CONCLUSIONES}

El cultivo de Cucurbita pepo var. 'Scallop' mostró gran adaptación a las condiciones agroclimáticas del centro de la Provincia de Buenos Aires, manifestado por su elevado rendimiento. 


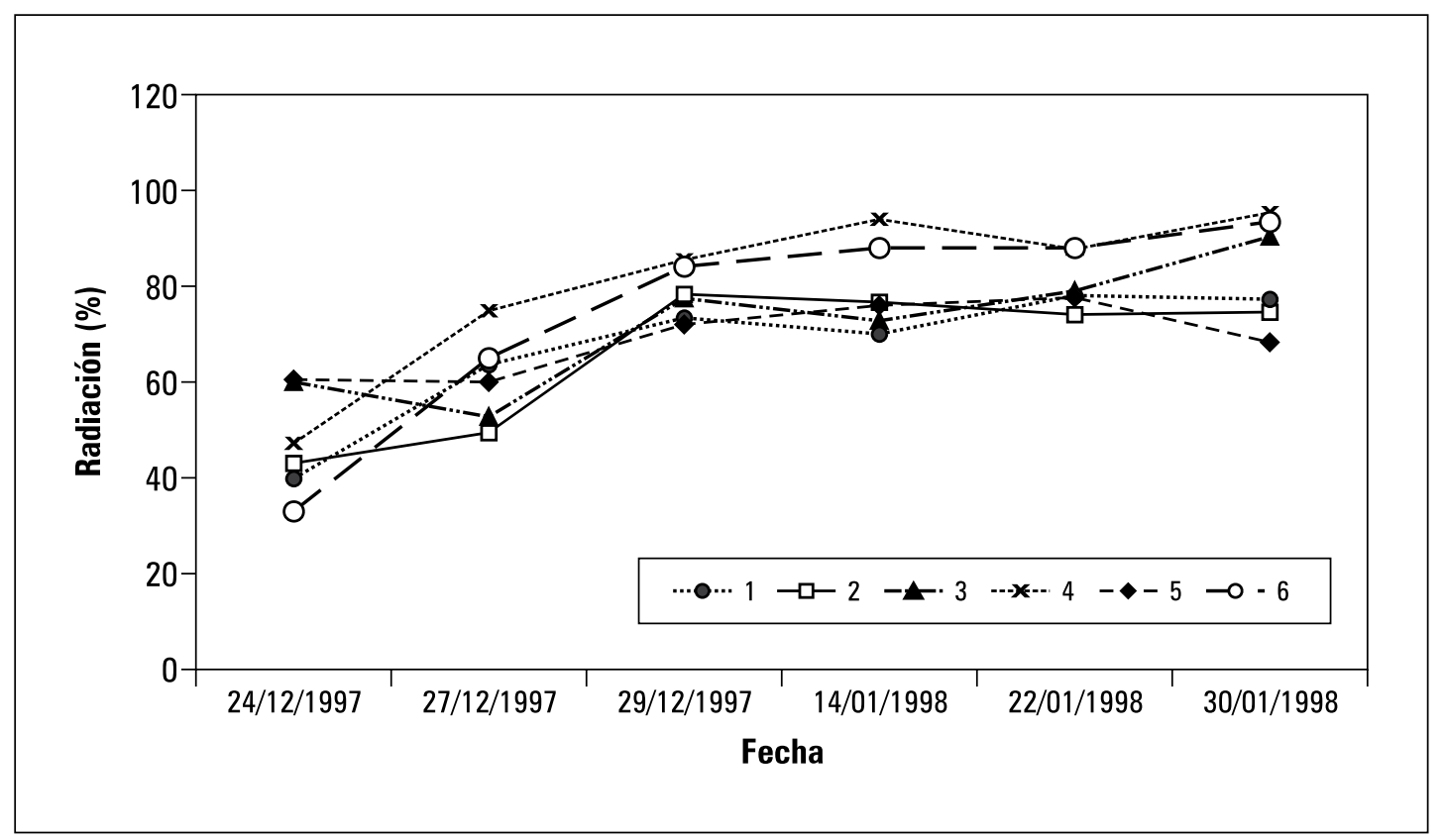

Figura 2. Porcentaje de radiación interceptada por el cultivo durante el período de cultivo.

Como en un ensayo anterior realizado en Cucurbita pepo 'Spaghetti' (Castagnino et al., 1998), las mediciones de intercepción de radiación efectuadas mediante el empleo de un medidor cuántico lineal ya indicaban durante la etapa de crecimiento vegetativo cuales serían los resultados a lograr a lo largo de las cosechas.

La aplicación del fertilizante fosfato diamónico permitió lograr un rendimiento de hasta $27 \%$ superior respecto del testigo. El empleo de todas las dosis probadas superaron al testigo sin fertilizar.

Este cultivo constituye una alternativa promisoria, novedosa, altamente productiva y muy atractiva que se adapta como cultivo ornamental y también productivo constituyendo una alternativa innovadora, principalmente orientada al mercado de las denominadas hortalizas 'Baby' que ha presentado un crecimiento potencial en el mercado en los últimos años, principalmente focalizado a un segmento muy importante integrado por niños y adolescentes. La difusión de esta hortaliza permitiría brindar un producto atrayente por su forma, de limitado aporte energético y alto valor nutricional, permitiendo brindar a la dieta magnesio, niacina y vitaminas A y C, características que permitirán, con un adecuado manejo, constituirlo en un alimento funcional. Es decir que la integración de dicha hortaliza a la dieta habitual permitiría contribuir en forma positiva a una o varias funciones del organismo, propiciando un mejor estado de salud y bienestar. 


\section{REFERENCIAS BIBLIOGRÁFICAS}

Alvarado, V. P. 1998. Efecto de diversos tipos de acolchados plásticos sobre la temperatura del sueloy su influencia sobreeldesarrollode malezas, precocidadyrendimiento del brócoli. Actas del IX Congreso Latinoamericano de Horticultura. Santiago, Chile. 275 p.

Cantamuto, M. 1997. Cultivo de melón con mulching en bandas angostas y herbicidas. Actas XX Congreso Argentina de Horticultura. Bahía Blanca, Pcia. de Buenos Aires, Argentina. 114 p.

Castagnino, A.; P. Sastre Vazquez y M. Navarro Dujmovich. 1998. Influenza di diverse tecniche colturali sulla intercettazione della radiazione e sulla resa di Cucurbita pepo var. Vegetable Spaghetti in condizioni di siccità. Actas XVIII Congreso Argentino de Horticultura, Termas de Río Hondo Pcia. de Santiago del Estero, Argentina. 54 p.

Castagnino, A.M.; P. Sastre Vázquez; K. Diaz; C. Boubee y F. Tognoni. 1999. Efecto de la restricción radical sobre el crecimiento y la arquitectura de plantines de pimiento Capsicum annum var. 'Frutescens'. Horticultura Brasileira 19(1), 1-4.

Castagnino, A.M.; P. Sastre Vázquez; A. Menet y S. Sasale, 2006. Evaluación de técnicas de defensa y de la densidad en el cultivo de una nueva hortaliza: Cucurbita pepo var. Vegetable Spaghetti. Revista Ciencias Agrarias $N^{\circ} 23$. En: http://www.uca.edu.ar/ esp/sec-fagrarias/esp/docs-revista/volumenes/tomo. php? numero=23_zapallo_spaguetti.

Castillo, H. 1998. Modificaciones estacionales de la temperatura del suelo por el uso de acolchados. Actas del IX Congreso Latinoamericano de Horticultura. Santiago, Chile. 229 p.

Darwich, N.A. 1994. Fósforo, un nutriente esencial para las plantas. Manual de fertilidad de suelos. INTA, Balcarce. 65 p.

Darwich, N.A. 1983. Niveles de fósforo asimilable en los suelos pampeanos. Idia 409/412, 1-5.
Darwich, N.A. 1984. Implantación y manejo del cultivo. En: Tecnología disponible para el cultivo de maíz en la zona Sudeste de la Pcia. de Buenos Aires. EEA Balcarce INTA 1, 9-17.

Darwich, N.A. 1989. Manual de fertilizada de suelos. SAGyP INTA ENICHEM Agricultura. Capítulos 4 y 5.

Emmert, E. 1957. Black polyethylene for mulching vegetables. Proc. Amer. Soc. Hort. Sci. 69, 464-469.

Giustiniani, L.; A. Graifenberg; L. Botrini y M. Curadi 2001. Salinitá e impianto nel allevamento dello zucchino. Rev. Colture Protette 30(3), 73-76.

Loy, B. y O. Wells. 1990. Effect of IRT mulches on soil temperature, early vegetative development in muskmelon and weed growth. Proc. Natl. Agricultural Plastics Congress 22, 19-27.

Martínez, R.S. 1997. Efecto de tres sistemas de manejo sobre la producción de zapallo tipo Butternut en lotes enmalezados. Actas XX Congreso Argentina de Horticultura. Bahía Blanca, Pcia. de Buenos Aires. Argentina. $114 \mathrm{p}$.

Mullins, C.; R. Allen Straw y A. Rutledge. 1992. Tomato production with fertigation and plastic mulch. Tenn. Farm Home Sci. pp. 23-27.

Paris, H. y H. Nerson 1986. Genes for intense fruit pigmentation of squash. American Genetics Association 77, 403-409.

Paris, H. 1988. Complementary genes for Orange Fruit Flesh Color in Cucurbita pepo. HortScience 23(3), 601-603.

Paris,H.1992.Arecessive, hypostaticgeneforplainlightfruit coloration in Cucurbitapepo. Euphytica 60(1), 15-20.

Quattrucci, M. 2001. Concimazione e qualità del cocotero. Revista Informatore Agrario 18, 75-79.

Trentini, L. 1996. Volume dell'alveolo e qualitá della piantina in vivaio. Italia. Vivaismo Orticolo, Suplemento L' Informatore Agrario 29(96), 47-62. 